\title{
MEDIO AMBIENTE
}

\section{BASURAS ESPACIALES UNA DIMENSIÓN NUEVA DEL DETERIORO AMBIENTAL}

\author{
SPACE DEBRIS. \\ A NEW FORM OF ENVIRONMENTAL DEGRADATION
}

*J Jairo Escobar R

\author{
Recibido Mayo 14-2014. Aceptado Julio 4-2014
}

\begin{abstract}
RESUMEN
Hace más de diez años la comunidad internacional comenzó a preocuparse por la seguridad del espacio extraterrestre y por el peligro que él representa para las misiones, comunicaciones e investigaciones y para el medio ambiente espacial circundante de la tierra, debido a las "basuras espaciales". Ellas son un tema altamente especializado, poco difundido y de limitado conocimiento dentro de la sociedad. Se conoce actualmente que la cantidad de basura que está orbitando el planeta se está incrementando, volviendo una realidad la posibilidad de que "llueva basura".

Debido a lo anterior, hemos considerado de importancia desarrollar el presente artículo a partir de una revisión de la literatura científica disponible en la red, en especial la provista por algunas agencias espaciales de Estados Unidas, Rusia, Francia, China, que monitorean desde 1990 el espacio exterior adyacente; y de la información publicada por el Comité para los Usos Pacíficos del Espacio Exterior de Naciones Unidas, UNCOPUS. REC 2014:20: 49-54
\end{abstract}

Palabras clave. Medio ambiente espacial, basuras espaciales

\section{¿Que son las basuras espaciales?}

Hay varias definiciones que se aproximan al problema de las basuras espaciales, algunos autores las definen como "restos, desechos, chatarra, o escombros" y tienen en común, tratarse de artefactos en desuso, satélites, cohetes o naves no operativas, restos de componentes de cohetes, como polvo, residuos de combustible de diversa naturaleza, partículas de pintura, restos de explosiones, colisiones y de choques de artefactos que

\begin{abstract}
SUMMARY
More than ten years ago the international community began to worry about the security of outer space and the danger he represents for missions, communications and research and to the space environment surrounding land, because "space debris". They are a highly specialized, little known and limited knowledge within society issue. It is now known that the amount of garbage that is orbiting the planet is increasing, becoming a reality the possibility that " rains garbage."

Because the above, we considered of importance to develop this article from a literature review Scientific available online, especially that provided by some space agencies of the United States, Russia, France, China, which monitor since 1990 adjoining outdoor space; and the information published by the Committee on the Peaceful Uses of Outer Space UNCOPUS
\end{abstract}

Key words. space environment, space debris

generan gran cantidad de escombros y de residuos que vagan por el espacio, muchos de ellos de pequeño tamaño, pero de un gran poder de destrucción. En esta definición se incluyen todos los objetos activos o inactivos que han sido lanzados o se han bajado de sus órbitas para ser hundidos en el mar, así como cohetes espaciales antiguos y en funcionamiento y otros objetos procedentes de la fragmentación de basuras o residuos en el espacio exterior.

\footnotetext{
* Ex asesor del Programa de Océanos y Áreas Costeras OCA/PAC, del Programa de las Naciones Unidas para el Medio Ambiente PNUMA- para los Planes de Accion: Pacifico Sudeste PSE, Gran Caribe, CAR y Pacifico Nordeste, NEP Correspondencia jjairoescobar@yahoo.com>
} 
Colectivamente la basuras espaciales se agrupan bajo el término de "Space Debris", definido por el Comité de Coordinación Interagencial sobre Basuras Marinas (Inter-Agency Space Debris Coordination Commitee, IACD) y por el Comité de las Naciones Unidas para el Uso Pacífico del Espacio Exterior (UNCOPUS) como "todo objeto fabricado por el hombre incluyendo fragmentos o elementos relacionados, ubicados en la órbita terrestre o que re-ingresan a la atmósfera y que no son funcionales".

Aunque se estima que la cantidad de todos esos desechos que vagan en el espacio exterior es baja y no representa un peligro inmediato, sí supone un riesgo para astronautas, para los trasbordadores espaciales, satélites y sondas espaciales activos. La NASA estima que la cantidad de basuras espaciales evolucionara en el tiempo en una cantidad más o menos estable hasta el 2055, fecha después de la cual, el número de objetos flotando en el espacio se disparará exponencialmente, en forma tal, que "salir de nuestro espacio exterior resultaría casi imposible" y el peligro que representa de que parte de la basura orbital, reingrese descontroladamente y precipite a la tierra sea una posibilidad cada vez más cercana"

De los objetos orbitando, existen aproximadamente 2,465 satélites artificiales, la mayoría de ellos están puestos en órbita terrestre baja (LEO), donde viajan alrededor de $27.400 \mathrm{~km} / \mathrm{h}$ (8 km/seg.) dando la vuelta al mundo cada 90 minutos. La principal excepción son los satélites de comunicación que requieren de órbita geoestacionaria (GEO)

\section{Antecedentes}

El interés por lo que hay fuera de la tierra en el espacio exterior siempre ha fascinado a la humanidad; existen registros antiguos de este interés, como "La Historia Verdadera" de Luciano de Samota (siglo II. de J.C.) y "Orlando El Furioso" de Ariosto (1532), ambas referencias con historias sobre la luna y más tarde Voltaire, con su cuento "Micromegas" (1752) que habla de la estrella Sirius. En 1870, disminuye la ficción y se acerca a la realidad, Verne escribe "Viaje al rededor de la Luna" (Wikipedia Org). Sin embargo, dentro del contexto de las basuras espaciales y/o chatarra espacial, sus antecedentes son consecuencia de dos empresas humanas conectadas con historiales convergentes en dos orígenes que juntos forman los antecedentes de la Ilamada Era Espacial. Uno hace referencia a los antecedentes históricos de los satélites espaciales, con fines pacíficos (como "The Brick Moon" en 1969, de E.E, Hale y "Los quinientos millones de la Begún" de Julio Verne, publicado en 1979), y otros con fines militares, de comunicaciones y de defensa estratégica. En 1983, el presidente de EE. UU, Ronald Regan, propuso dentro de la conocida "Guerra de las Galaxias", utilizar los sistemas balísticos utilizados en tierra y en el espacio a fin de defender los Estados Unidos de los misiles balísticos intercontinentales. Dentro de este contexto, se inició una militarización del espacio exterior colocando en órbita un número no conocido de satélites espías y otros objetos espaciales, y dejando el espacio exterior lleno de chatarra espacial (Reagan. 1982; Wikipedia Org op cit). Con el fin de la guerra fría, con el colapso del bloque soviético y con el fin de la URSS, se redujo la militarización del espacio pero se incrementó notablemente el número de satélites para comunicaciones y para otras aplicaciones pacíficas, continuando con la chatarreterizacion del espacio exterior.

Después del lanzamiento del satélite ruso Sputnik, en 1957, hasta la fecha se han registrado, más de 35.000 objetos artificiales en las órbitas de la tierra. La Red de Vigilancia Espacial (SSN), ha rastreado, más de 26.000 objetos orbitando y mantiene vigilancia a más de 8,000 objetos de fabricación humana. Algunos de estos objetos han regresado a tierra donde se desintegran al contacto con la atmósfera y hay ejemplos de otros que eventualmente han llegado a la superficie terrestre, como los fragmentos del satélite soviético Comos 2251 en Canadá, en 1978. En los pocos casos conocidos de regreso, no hay reportes conocidos de heridos por basura espacial precipitada a tierra. Los expertos señalan que actualmente el riesgo para los habitantes es pequeño, pues la mayoría cae al océano (ejemplo, chatarra del "Fobus -Grunt en el Océano Pacifico,- 300 kilos de chatarra del satélite de la NASA -UARS, en el Pacifico-, chatarra del satélite alemán ROSAT, en el Golfo de Bengala, etc.). La gran mayoría de la basura espacial queda 
inactiva dando vueltas alrededor del planeta, por lo que hoy hay orbitas más densamente pobladas de satélites, como aquellos ubicados a una altitud de 700 kilómetros. Otros, como los de la constelación GPS, mucho más altas a 20.000 Kilómetros pueden ser importantes y demandan atención.

\section{¿Cuáles son las fuentes y cuánta basura espa- cial hay?}

Aproximadamente el $60 \%$ de los objetos (chatarra espacial), ha sido catalogado como fragmentos de naves espaciales y fragmentos de cohetes o etapas de cohetes. A menudo la fragmentación resulta de una explosión accidental producida por escape de combustible espacial o de otros agentes químicos reactivos contenidos en las etapas de los cohetes impulsadores. Esta es la primera fuente de fragmentación productora de desechos -space debris-. Las colisiones entre objetos de gran tamaño (generalmente cohetes y naves espaciales) y otras piezas de basuras constituyen otra fuente adicional de basuras espaciales. Otra fuente significativa de basuras espaciales la constituye la separación de material y de objetos de los satélites durante su puesta en órbita o durante su operación, tales como, lentes y material de escudos de protección o coberturas, mangueras y otro material que se puede separar eventualmente de los satélites y orbitar descontroladamente. Las basuras relacionadas con las misiones espaciales y con las etapas de los cohetes propulsores de lanzamiento que se mantienen en órbita, represen-

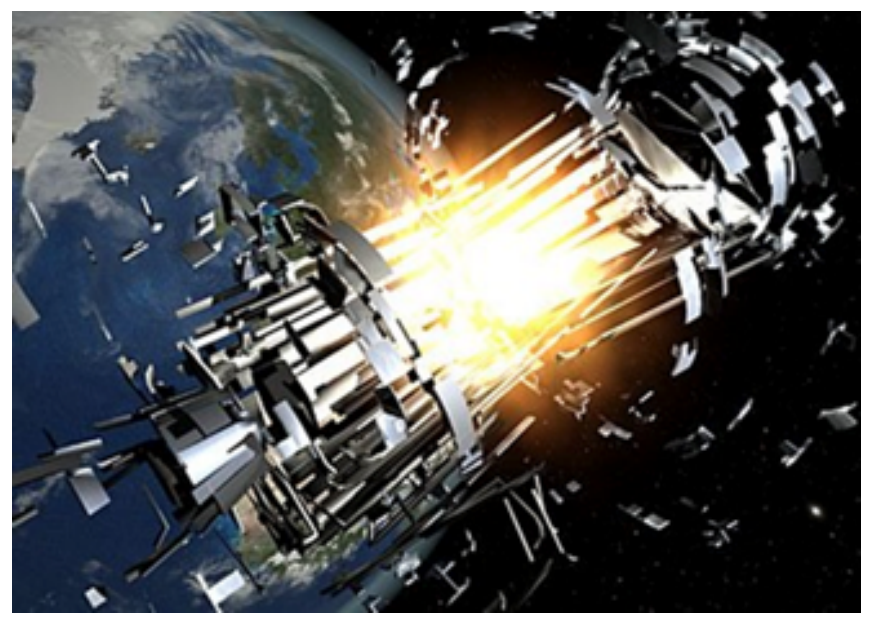

Producción de basura, por la explosión de una nave, cohete. Disponible en: http://factoides.com.ar/post/1269471060/basura-espacial\#axzz1n3iahmzg. si tan cerca del $18 \%$ de las basuras espaciales catalogadas. Los satélites no operativos constituyen el $15 \%$ de estas basuras catalogadas. Además, existen casos de destrucción deliberadas de satélites que contribuyen de forma significativa a incrementar la cantidad total de basuras espaciales sobre las cuales no hay registros.

De acuerdo con la Agencia Espacial Europea (AEA), la composición de los objetos artificiales que orbitan la tierra es aproximadamente:

\begin{tabular}{|l|c|}
\hline \multicolumn{1}{|c|}{ Basura/chatarra } & $\begin{array}{c}\text { Cantidad estimada en la } \\
\text { órbita terrestre (\%) }\end{array}$ \\
\hline Naves operativas & 7 \\
\hline Naves obsoletas & 22 \\
\hline Restos de cohetes & 17 \\
\hline $\begin{array}{l}\text { Objetos relacionados } \\
\text { con misiones espacia- } \\
\text { les }\end{array}$ & 13 \\
\hline Otros fragmentos & 41 \\
\hline
\end{tabular}

Tomado de Basura Espacial, disponible en- http://es.wikimedia.org/wiki/Basura_espacial.si

Recientemente, un viejo satélite espía de EE. UU. EI "USA 193", con un peso de 9,072 kilos encendió la alarma, quedando fuera de control y perdiendo altura (Piña, 2008). En la primavera de 2009, el choque de dos satélites artificiales (el Cosmos 2251 de Rusia, y el Iridium 33, de Estados Unidos) contribuyó a elevar el número de desechos mayores de 10 centímetros. Todas estas colisiones se produjeron en la región de la órbita baja, LEO (Naciones Unidas, 2009). Otras fuentes con aportes menores provienen de los accidentes y de las colisiones durante las operaciones en órbita, así como del abandono en el espacio después del periodo de vida útil de satélites y otros objetos espaciales que no se pueden regresar

La estadística anterior muestra significativamente que entre 18 de las 25 fragmentaciones no deliberadas involucraron cohetes de propulsión y la explosión de los residuos de los propelentes dejados a bordo en estos cohetes. También se ha observado que las colisiones entre los objetos que orbitan cercanos unos de otros (orbitan cerca de 5 kilómetros uno de otro), han llegado a ser muy frecuentes 
en la órbita baja, LEO, duplicándose entre 2006 y 2009. La mayoría de estas basuras espaciales orbitan a velocidades entre 3 kilómetros/segundo y 7,7 kilómetros/segundo, por lo que el riesgo de una colisión tendrá serias consecuencias. La mayor parte de las basuras espaciales que están concentradas en esas áreas del espacio que son de importancia y utilidad para el desarrollo de la humanidad. Entre 1957 y 1996, los Estados Unidos y Rusia agregaron al catálogo de objetos espaciales un promedio de 100-120 objetos/año y entre 1996 y 2006, los Estados Unidos adicionó un promedio de 16 objetos/año, mientras que Rusia hizo lo propio con un promedio de 44 objetos/año (Piña, 2008)

\section{Tamaño de la basura espacial}

Se reporta que hay orbitando la tierra más de 21.000 objetos fabricados por el hombre mayores de 10 centímetros de tamaño y se estima que existen adicionalmente entre 400,000 y más de 600,000 objetos de tamaño entre uno y diez centímetros en la órbita baja LEO, y cientos de millones de objetos de tamaño entre un centímetro y un milímetro. Además, se han catalogado entre 14,000 y 15,800 objetos mayores de diez centímetros en la órbita baja LEO. Especialmente, han sido identificados en relación con un lanzamiento específico o con un evento de liberación. De estos, solo entre el $6 \%$ y el $7 \%$ son satélites operativos; lo que significa que al mínimo más del $90 \%$ de los objetos ubicados en las orbitas terrestres son objetos no controlados de cualquier tamaño. Por comparación, se ha estimado que la cantidad total de basuras espaciales que mide entre uno y diez centímetros y que orbita en todas las latitudes es aproximadamente de 750,000 objetos, mientras que la cantidad de basura espacial mayor de diez centímetros se aproxima a los 24,000. Conforme a estos estimativos, se cree que ligeramente la mitad de todas las basuras espaciales mayores de un centímetro se ubican en la órbita baja, LEO. El $57 \%$ de estas basuras han sido catalogadas como provenientes de la desintegración de objetos espaciales, 33\% provenientes de cargas útiles, $11 \%$ cohetes de empuje $\mathrm{y}, 11 \%$ tienen origen en misiones espaciales.

Todos los objetos que no están activos en las ór- bitas terrestres pueden ser: satélites, pedazos de ellos, resultados de explosiones, hasta basura dejada por un astronauta. Se identifica como la fuente de mayor ingreso de basuras espaciales a las explosiones de las últimas etapas de los cohetes de propulsión en las capas superiores. Antes de 2007, la principal fuente de basura espacial la constituía las etapas de cohetes de propulsión en desuso. Pero con la destrucción intencional, en una órbita de baja altitud -865 kilómetros-, del satélite meteorológico Feng Yun-1C, de China, con un misil balístico de rango medio, se elevó en Enero de 2007 , la cantidad de basuras espaciales.En una décima de segundo quedo convertido en 2.000 fragmentos de tamaño entre 5 y 10 centímetros, 35.000 fragmentos de alrededor de un centímetro y cerca de un millón de pedazos menores de un milímetro-, "En ese instante, el volumen de los desechos espaciales que orbitan la tierra se incrementó en cerca de 15\%" (Piña, 2008). Ese mismo año, en febrero, una etapa (tipo Briz-M) de un cohete ruso Protón, lanzado hacia un año, después de una operación fallida y en órbita con todo su combustible, exploto debido a la elevada temperatura producida por el rozamiento y un millar más, de grandes fragmentos, de entre 1 y 10 centímetros, quedaron dando vuelta a la tierra (Piña, op.cit).

\section{Basura Espacial y Satélites en Órbita}

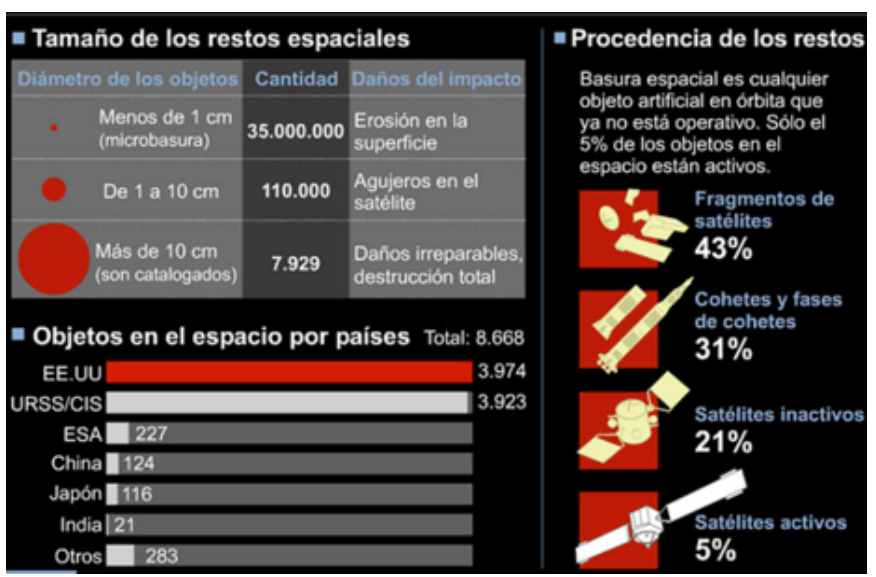

Tomado de: José Mª Piña 2008 "Basura Espacial y Satélites en Órbita, en "A día de hoy" 07/02/2008 en http://www.astropractica.org/tact/basesp/basesp. $\mathrm{htm}$ si

Los 10 accidentes espaciales más generadores de basura 


\begin{tabular}{|l|c|c|c|c|c|}
\hline Nombre común & $\begin{array}{c}\text { Año del } \\
\text { accidente }\end{array}$ & $\begin{array}{c}\text { altitud-kilóme- } \\
\text { tros }\end{array}$ & $\begin{array}{c}\text { Cantidad de basu- } \\
\text { ras catalogadas }\end{array}$ & $\begin{array}{c}\text { Cantidad de basu- } \\
\text { ras ingresadas a } \\
\text { orbita }\end{array}$ & Causa del accidente \\
\hline Feng-Yun 1C & 2007 & 850 & 2.841 & 2.756 & Colisión Intencional \\
\hline Cosmos 2251 & 2009 & 790 & 1.267 & 1.215 & Colisión accidental \\
\hline $\begin{array}{l}\text { Etapa 2 de Cohete } \\
\text { propulsor }\end{array}$ & 1996 & 625 & 713 & 63 & Explosión accidental \\
\hline Iridium-33 & 2009 & 790 & 521 & 498 & Collision accidental \\
\hline Cosmos 2421 & 2008 & 410 & 509 & 18 & Desconocida \\
\hline $\begin{array}{l}\text { SPOT 1 Cohete } \\
\text { propulsor }\end{array}$ & 1986 & 805 & 492 & 33 & Explosion accidental \\
\hline $\begin{array}{l}\text { Cohete propulsor } \\
\text { OV2-1/LCS 2 }\end{array}$ & 1965 & 740 & 473 & 36 & Explosion accidental \\
\hline $\begin{array}{l}\text { Cohete propulsor } \\
\text { Nimbus 4 }\end{array}$ & 1970 & 1075 & 374 & 248 & Explosion accidental \\
\hline $\begin{array}{l}\text { Cohete propulsor } \\
\text { TES }\end{array}$ & 2001 & 670 & 370 & 370 & Explosion accidental \\
\hline $\begin{array}{l}\text { Cohete propulsor } \\
\text { CBERS 1 }\end{array}$ & 2000 & 740 & 343 & 189 & Explosion accidental \\
\hline & & 7903 & 5172 & & \\
\hline
\end{tabular}

1/ a mayo 2010

2/De: Ten Satellite Breakups16 "Orbital Debris Quarterly Newsletter", NASA Orbital Debris Program Office, Vol. 14 Issue 3 , July 2010 , online at www.orbitaldebris. jsc.nasa.gov/newsletter/pdfs/ODQNv14i3.pdf. si

\section{¿Dónde se localizan las basuras espaciales?}

La distribución de las basuras espaciales y de los satélites operativos no es uniforme y está en función de las actividades humanas en el espacio. La mayor parte de las basuras están concentradas en órbitas de mayor utilidad para el hombre, donde se concentra la mayor actividad espacial humana, particularmente en LEO, entre los 600 y 1,500 kilómetro, allí se ubica la mayoría de los satélites de observación de la tierra, al igual que en la órbita geoestacionaria GEO, a 36,000 kilómetros, donde se localizan la mayoría de los satélites de telecomunicaciones (Liuo \& Jhonson, 2006). En general, la mayor parte de esta basura espacial se sitúa actualmente en las órbitas más transitadas por satélites artificiales - de comunicaciones, militares y astronómicos-, como lo es la órbita baja LEO, a altitudes entre 800 y 1,200 kilómetros, lo que da como resultado una muy alta probabilidad de colisión en esta órbita. Las máximas densidades se ubican a altitudes de $\pm 850, \pm 1,000, \pm 1,000, \pm$ 2,000 y $\pm 36,000$ kilómetros (Wikimedia Org).

Aproximadamente el $7 \%$ de los objetos espacia- les (cerca de 560 satélites, de ellos, 381 bajo control conocido), están en funcionamiento, el resto constituye chatarra espacial. Se ha informado que cerca de 100 toneladas de fragmentos generados por aproximadamente 200 explosiones están en órbita, concentrados principalmente en la órbita baja de la Tierra LEO, y alguna parte más allá de la órbita geoestacionaria GEO. Los desechos espaciales ocupan cada vez más espacio entre los objetos que orbitan la Tierra (Liuo \& Jhonson, 2006).

Pero no solo hemos contaminado nuestras orbitas espaciales con basura espacial sino que también nuestros desechos y chatarras y equipo en desuso lo estamos esparciendo en otros lugares de nuestra galaxia. Hemos dejado en Marte 8,053 kilos de material espacial, que corresponden a sondas espaciales, robots teledirigidos y artefactos de prospección, sin incluir otro material proveniente de los vehículos nodriza, tales como escudos térmicos ruedas etc. También hemos depositado en Venus 22,000 kilos de herramientas abandonadas que constituyen despojos y testigos mudos de nuestro afán de expandir nuestras fronteras. 


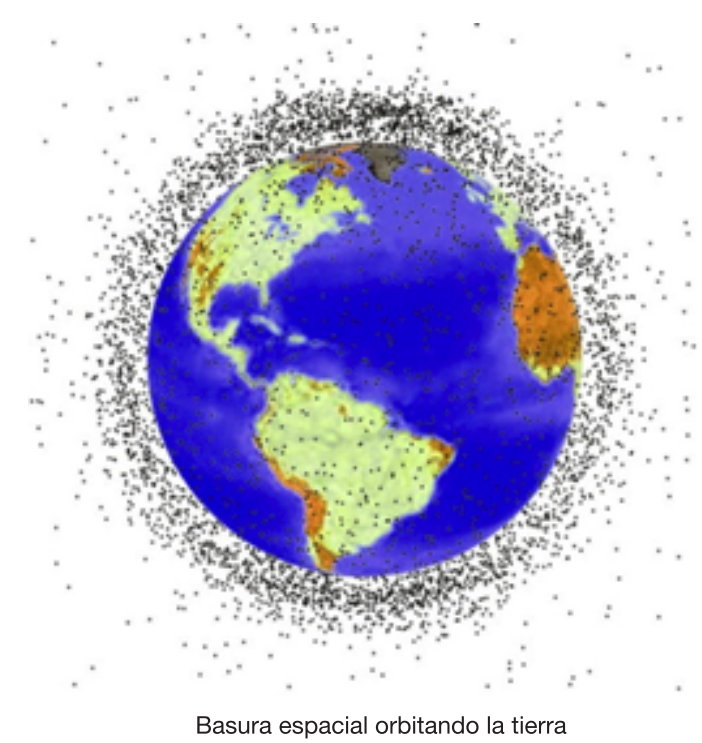

Fuente:http://factoides.com.ar/post/1269471060/basura-espacial\#ixzz1n3imkVN3 SI

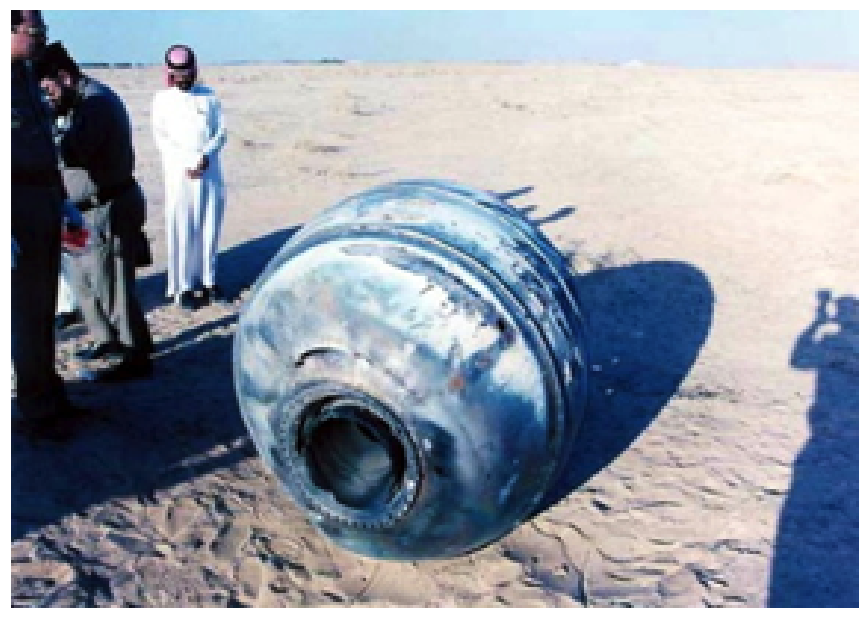

Chatarra espacial- Precipitada en el Desierto de Sahara Fuente:http://factoides.com.ar/post/1269471060/basura-espacial\#ixzz1n3imkVN3 SI

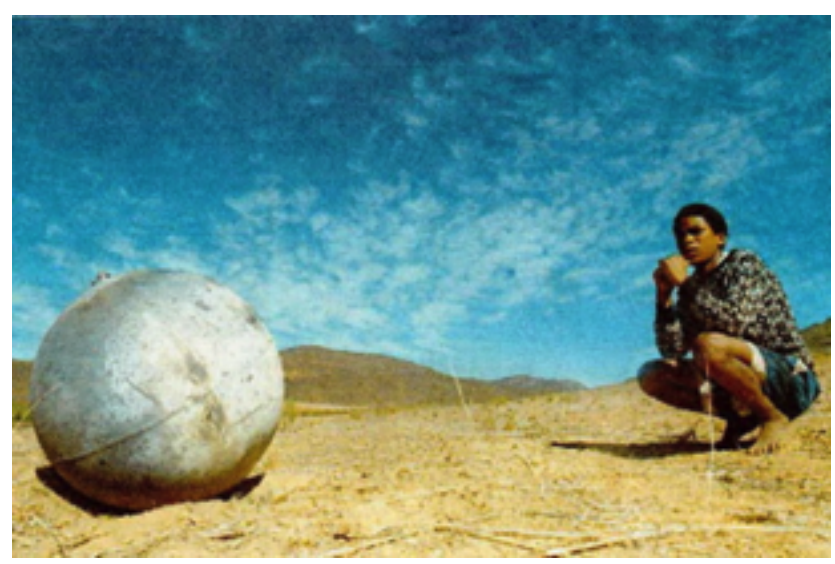

Chatarra espacial- Precipitada en Africa

Fuente:http://factoides.com.ar/post/1269471060/basura-espacial\#ixzz1n3imkVN3 SI

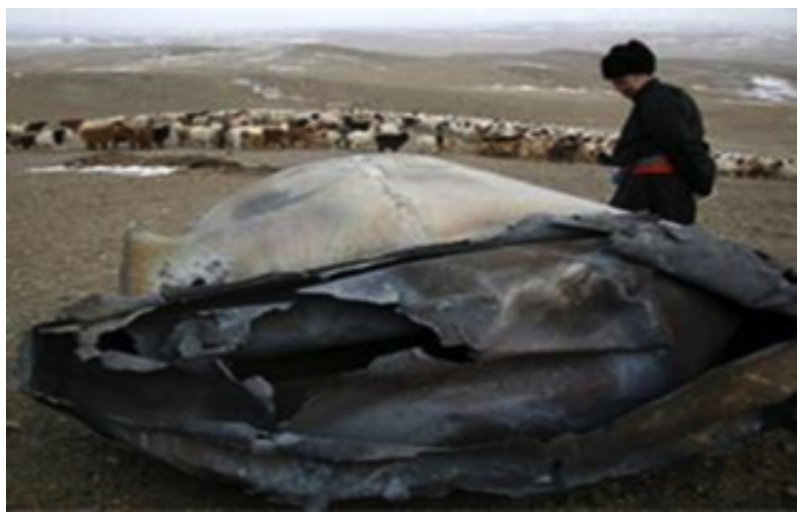

Chatarra espacial precipitada en Mongolia Fuente:http://factoides.com.ar/post/1269471060/basura-espacial\#ixzz1n3imkVN3 SI

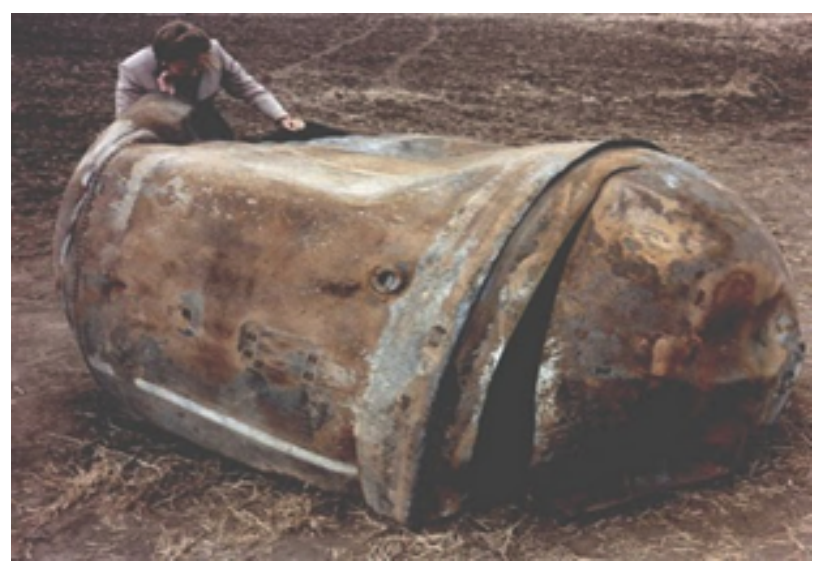

Chatarra espacial caída en la Provincia de Mendoza (Argentina) Fuente:http://factoides.com.ar/post/1269471060/basura-espacial\#ixzz1n3imkVN3 S

\section{Las tendencias en la cantidad de basuras es- paciales}

La basura espacial muestra una tendencia a incrementarse. Para el año 2011 la Oficina de la NASA sobre Restos Orbitales informó un incremento de $1,4 \%$ con respecto a los niveles del 2010 . Esto es, hay 218 objetos más orbitando alrededor de la Tierra, una cifra que parece aumentar año tras año. Estimó además, que hay actualmente orbitando la Tierra, 16,177 escombros, desde cohetes y etapas de lanzamiento intactos hasta restos de estos aparatos dañados. Por otro lado, NASA mide cada trimestre el volumen de los objetos que orbitan la Tierra. De esta forma, hasta el 31 de marzo del año 2011 se contabilizaron 15,855 objetos; para junio la cifra aumentó a 16,094 y para diciembre fue de 16.117 (Oficina de la NASA sobre Restos Orbitales). 
Nómina de los países lanzadores y países aportantes de basura espacial

La "Commonwealth of Independent States (CIS)" -Reino Unido y sus colonias-, se registra como la mayor aportante de basuras espaciales, con total de 6.087 objetos, seguido de Estados Unidos con 4.850 y de China con 3,615. La Comunidad Europea (Agencia Espacial Europea ESA), constituye el menor aportante de desechos espaciales del grupo de países aportantes (Grupo de Lanzadores), con tan solo 85 objetos en el 2011, de los que concretamente 41 proceden de explosiones y 44 son cohetes, restos de cargas útiles y demás escombros. Además, hay países aportantes, por fuera de la Agencia Espacial que envían y "tiran a la órbita terrestre" basuras espaciales, como es el caso de Francia, con 489 objetos, Japón 189, e India con 176 (Oficina de la NASA sobre Restos Orbitales).

\section{Detección y Monitoreo de las Basuras Espacia- les}

En la órbita baja terrestre LEO, (típicamente definida como la de altura inferior a los 2.000 kilómetros), el trayecto de las basuras son detectadas por barrido de radar. A alturas por debajo de 600 kilómetros, el recorrido de los objetos mayores de 10 centímetros son detectados con estos barridos, pero a medida que se incrementa por encima de 5,000 kilómetros a altura de la órbita, el tamaño límite de la basura que puede detectar el radar decrece a más de un metro. La detección y el recorrido de los objetos espaciales y/o basuras espaciales que se encuentran en la órbita geoestacionaria GEO (aproximadamente a 36,000 kilómetros) y en otras orbitas altas, generalmente se realiza con telescopios ópticos y con grandes y potentes mecanismos de radar.

\section{El problema de las basuras espaciales}

Los satélites que orbitan alrededor de la Tierra, una vez que finalizan sus ciclos de vida útil y no son recuperados, se convierten en basura espacial que vaga sin control, y puede llegar a caer poniendo en riesgo a la población en tierra. Además, hacen peligrar las exploraciones espaciales que están en marcha, por el riesgo de que alguna nave colisione con los satélites abandonados. Desde 1991, se han registrado tres colisiones en la órbita terrestre debido a las basuras espaciales. Estas colisiones irán en aumento y producirán más desechos en órbita debido a que, con los adelantos tecnológicos cada año ingresan a la órbita terrestre un número mayor de objetos espaciales con diferentes propósitos y muy pocos de estos regresan a tierra, de tal forma, que existe un factor acumulativo de estos objetos lo que hace que el riesgo de un accidente o una colisión sea alto.

Cualquier impacto entre dos objetos espaciales de masa importante, crearan una basura adicional de metralla de chatarra espacial resultante de la fuerza de colisión. Cada pedazo de metralla tiene el potencial de causar un daño adicional en otros objetos orbitando, semejante a una reacción en cadena, creando más basura espacial. Con una colisión bastante grande (entre una estación espacial y un satélite, por ejemplo) la cantidad de basura generada "podría ser lo suficientemente alta para hacer la órbita baja de la tierra inutilizable" (Kessler, 1991).

\section{Consecuencias.}

1. Toda esta basura impide vuelos más lejanos a los $600 \mathrm{~km}$ de altura. Las naves actuales, como la Estación Espacial Internacional, orbitan bastante por debajo de ese nivel, a $364 \mathrm{~km}$.

2. La cantidad de chatarra espacial seguirá creciendo sin control, y muchos de los objetos se alejarán lentamente y otros caerán a la Tierra, como ya ha sucedido en varias oportunidades. Los cálculos predicen que para el año 2055, será imposible lanzar cualquier misión espacial sin toparse con uno de estos objetos, a menos que se desarrolle y ejecute un plan para librarse de ellos.

3. De acuerdo con el resultado de ejercicios de simulación, se estima que las causas principales de las futuras colisiones serán las explosiones importantes del fuselaje de cohetes y satélites gastados ubicados en la órbita LEO. La gran mayoría de los satélites activos que orbitan en la GEO, tienen posiciones bien definidas con respecto al ecuador terrestre. Sin embargo, estos satélites deben realizar maniobras frecuentes para no sobrepasar los límites de las posiciones que les han sido asignadas, pues cualquier objeto pasivo en esa región puede desviarse de esas posiciones y chocar con cualquiera de los 


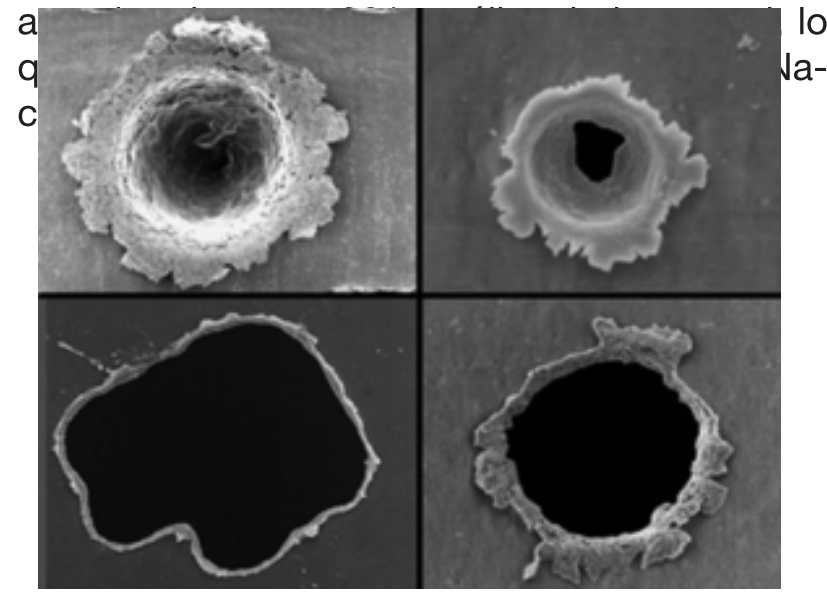

Impactos de la basura espacial sobre vehículos y satélites espaciales SI

Contexto jurídico internacional para las basuras espaciales

En 1963 la Asamblea General de las Naciones Unidas aprobó la Declaración de Principios Jurídicos que deben regir las Actividades de los Estados en la Exploración y Utilización del Espacio Ultraterrestre. En desarrollo de esos principios se han originado cinco Convenios Internacionales multilaterales, que constituyen el marco jurídico actual "Juris Spatiales Internationalis" para el tratamiento de las actividades, exploración, utilización y ocupación con fines pacíficos del espacio exterior. Un Convenio Marco y cuatro instrumentos suplementarios, constituyen el contexto jurídico para las basuras espaciales, además existen guías y declaraciones no vinculantes que versan sobre las basuras espaciales:

- El Tratado sobre las Actividades de los Estados en la Exploración y Utilización del Espacio Ultraterrestre, incluso la Luna y otros Cuerpos Celestes (TEU.1967) aprobado el 29 de noviembre de 1966;

- Acuerdo sobre el Salvamento y la Devolución de Astronautas y la Restitución de Objetos Lanzados al Espacio Ultraterrestre-Acuerdo de Salvamento (ASDA, 1968), aprobado el 19 de diciembre de 1967; Convenio sobre Responsabilidad Internacional por Daños Causados por Objetos Espaciales- Convenio Sobre Responsabilidad (RES-1972), aprobado el 29 de noviembre de
1971;

- Convenio sobre el Registro de Objetos lanzados al Espacio Ultraterrestre- Convenio sobre Registro, (REG-1975) aprobado el 12 de noviembre de 1974 y;

- Acuerdo que debe Regir las Actividades de los Estados en la Luna y otros Cuerpos CelestesAcuerdo de la Luna (LUNA -1979) aprobado el 5 de diciembre de 1979;

En la actualidad no existe una regulación internacional sobre el control de la "situación del tráfico espacial" . El Tratado Internacional sobre el Espacio Exterior, establece que "todos los Estados tienen libre acceso al espacio, a su exploración y uso pacífico". No obstante, no existe ningún mecanismo de control aceptado globalmente para monitorear las maniobras de satélites y cambios de órbitas. Un artículo, "Space Debris- A Threat to Modern Society" de los científicos Suecos (Andersson Ch, Rosenquist L, Persson M y E. Bernhardsdotter, 2011, Publicado en Strategic Outlook en 2011), pone de manifiesto la necesidad de un debate internacional para una nueva reglamentación internacional y de más investigación para mantener limpia el área del espacio de interés para el desarrollo y el progreso de la humanidad.

Tampoco existe una obligación internacional para mitigar el riesgo asociado con las basuras espaciales, pero por el Tratado Internacional sobre el Espacio Exterior, los Estados están "obligados a evitar la contaminación peligrosa" del ambiente del espacio exterior y para adelantar "las consultas necesarias apropiadas cuando se considere que existen razones para creer que una actividad planeada para ser realizada en el espacio exterior pueda potencialmente causar un daño peligroso o interferencia con otro Estado", Tampoco hay claridad sobre la existencia de la obligatoriedad legal para intercambiar información con otros Estados-Actores Espaciales, con el propósito de evitar choques o colisiones. Sin embargo, el Tratado, ratifica la obligación del Estado de "realizar consultas con respecto a interferencias potenciales peligrosas con otros.

Existe aún una amplia discusión respecto a la asignación de responsabilidades con respecto 
al daño resultante por las basuras espaciales. El Convenio Internacional para la Responsabilidad del Daño producido por Objetos Espaciales de 1972, permite que la responsabilidad puede establecerse por vía de dos sistemas: fallas basadas en la responsabilidad por daños causados en el espacio, y responsabilidad absoluta por daños causados en la Tierra. Sin embargo, existe dificultad tanto para establecer fallas y causales cuando dos objetos en el espacio colisionan (Black \& Butt. 2002).

Para mitigar el efecto de las basuras espaciales, las Naciones Unidas, a través de su Comité para el Uso Pacífico del Espacio Exterior UNCOPUOS, adopto en el 2007 la propuesta del Comité de Coordinación Interagencial sobre Basuras Marinas- Inter-Agency Space Debris Coordination Commitee, (IACD), de las Guías para la Mitigación de Basuras Espaciales (UNCOPUOS-Space Debris Mitigation Guidelines, IACD 2002), (Resolución 62/217 del 22 de Diciembre del 2007). Las Guías UNCOPUOS, están agrupadas en siete guías básicas que contienen recomendaciones generales para ser implementadas por los Estados, primeramente a través de las legislaciones nacionales, regulaciones y/o directivas políticas.

Son de implementación voluntaria y constituyen el primer paso para garantizar el uso sostenible a largo plazo del medio ambiente del espacio exterior inmediato. El propósito general de las guías es limitar la generación de basuras espaciales en el medio ambiente. Las Guías se aplican tanto en la fase de planeación de las misiones espaciales, como en el diseño y operación de vehículos y objetos espaciales y en los etapas orbitales que serán inyectadas en la órbita terrestre (Black \& Butt, 2002).

\section{¿Cómo se prevee el retiro y eliminación de la chatarra espacial?}

Actualmente gran parte de los esfuerzos se enfocan en retirar objetos grandes, como satélites en desuso. Se plantea enviarlos a zonas menos saturadas o bien arrastrarlos hacia la atmósfera para que se incendien y destruyan. Para efectos de los retiros y reingresos, los desechos se han agrupado en dos: Los peligrosos, que son los que tienen una alta probabilidad de alcanzar la superficie te- rrestre, y los menos peligrosos, cuya posibilidad de reingresar a la superficie terrestre es baja o no van a llegar porque pueden desintegrarse o explotar antes de lograrlo.

Se conoce que, el tratamiento directo de las basuras espaciales (limpieza) es muy difícil y complicado con la tecnología actual y por las condiciones de altas velocidades y el pequeño tamaño de la metralla espacial, que hace que la recuperación y eliminación sea difícil y altamente improbable. La mayoría de la basura en la órbita baja de la Tierra sucumbiría eventualmente a la resistencia del aire en la alta atmosfera, pero su proceso requeriría cientos de miles de años. Si esta basura fuera susceptible magnéticamente, podría caer, debido a la fricción con el campo magnético de la tierra pero esto tomaría decenios (Wikimedia Org.)

A efectos de reducir el mínimo la posibilidad de dañar otros vehículos espaciales, las nuevas misiones se diseñan de forma que los vehículos o satélites sean desechables en forma segura al final de su vida útil, por medio de una re-entrada controlada en la atmosfera para el caso de orbitas bajas o el ascenso a una órbita cementerio en el caso de ocupar una órbita geoestacionaria. La fricción del aire en las órbitas más bajas mantiene las zonas limpias. Para altitudes por debajo de 500 kilómetros, los objetos serán barridos en cuestión de meses. En caso de la órbita geoestacionaria muy poblada de satélites, esto no ocurrirá, por lo que los satélites viejos se suben a órbitas más altas donde no se pueden cruzar con los que están en órbita geoestacionaria a fin de preservar esta última (Wikimedia Org.)

Actualmente se está estudiando el costo-beneficio de las hipótesis plausibles de reducción de los desechos, sobre la base del proyecto "Servicio de extremo a extremo sobre desechos espaciales", de 2004-Los métodos específicos para retirar de las orbitas la chatarra u objetos de mayor tamaño tiene como fin prevenir la fragmentación en las órbitas de uso más frecuente. Para retirar esas chatarras u objetos se pasarán de su órbita a la atmósfera o por medios diferentes, se confinarán en una órbita de eliminación. Para cambiar las órbitas de esos objetos o chatarra, se propone 
el uso de robots con sistemas convencionales de propulsión o el despliegue de estructuras grandes para interactuar con la atmósfera terrestre (aumento de arrastre) con el campo geomagnético (lazos electrodinámicos, velas magnéticas) o con la presión del viento solar (velas solares).
Aunque se reconoce la importancia de la retirada de los desechos espaciales, todavía no se ha demostrado la viabilidad ni la eficacia de los métodos propuestos. En común, las propuestas actuales para retirar desechos grandes parecen ser técnicamente exigentes y costosos. Las propuestas para eliminar objetos pequeños tienen como

fin la desviación directa o la descomposición térmica mediante sistemas laser, estas también están aún en un nivel de experimentación

\section{BIBLIOGRAFIA}

1. Andersson Ch., Rosenquist. L, Persson M, y E. Bernhardsdotter, (2011): "Space Debris- A Threat to Modern Society" en Strategic Outlook, 2011- Disponible en: http://news.gc.ca/web/articleen.do?ctr.sj1D=\&mtd=tp\&ctr.mnthndVl=\&nid=575749\&crtr. dpt1D=yr

2. Black S \& Youssef Butt, (2002:, "The Growing Threat of Space Debris”, online at http://bos.sagepub.com/content/66/2/1.full. D.J, Kessler; (1991);."Collisional Cascading: The Limits of Population Growth in Low Earth Orbit" Advances in Space Research 11: pp. 63, disponible en: http://www.sciencedirect.com/science/article/pii/027311779190543S

3. Dan C, s.f. www.ojocientifico.org/users/carlos-dan.

Inter-Agency Space Debris Coordination Commitee, IACD; (2002):, Space Debris Mitigation Guidelines, IACD, 02-01, 15 October 2002, disponible http://stage.tksc.jaxa.jp/spacelaw/kokusai_utyu/space_debris/iadc.pdf

4. King, Hele, D. G (1959); “Analysis of the Orbits of the Russian Satellites”, Proc. Roy. Soc. London. A. 253, 529-538pp, 1959.

5. Liou J. -C. \& Johnson N. L, et al (2996):.Science, 311. $340-341$

7. National Aeronautics and Space Administration NASA, National Space Center Data (2012):, Sputnik-1, October/21/2012NSSCD-ID-1957.001B,disponiblen http://nssdc.gsfc.nasa.gov/nmc/spacecraftDisplay.do?id=1957-001B

NASA Orbital Debris Program Office, (2010): “Ten Satellite Breakups16 “Orbital Debris Quarterly Newsletter", NASA Orbital Debris Program Office, Vol. 14 Issue 3, July 2010, online at www.orbitaldebris.jsc.nasa.gov/newsletter/pdfs/ODQNv14i3.pdf. Oficina de la NASA sobre Restos Orbitales- Escombros Espaciales, En: http://www.vigoalminuto.com/2012/01/22/16-117-escombros-espaciales-orbitan-ya-alrededor-de-la-tierra/

8. Naciones Unidas, Comité para los Usos Pacificos del Espacio Exterior UNCOPUOS; (2012): "Long-term sustainability of outer space activities Active Debris Removal - An Essential Mechanism for Ensuring the Safety and Sustainability of Outer Space" A

9. Report of the International Interdisciplinary Congress on Space Debris Remediation and On-Orbit Satellite Servicing Organizers- Institute of Air \& Space Law, McGill University, Montreal, Canada Institute of Air \& Space Law, Cologne, Germany, International Association for the Advancement of Space Safety, Noordwijk, The Netherland, Document Committee on the Peaceful Uses of Outer Space Scientific and Technical Subcommittee, Forty-ninth session, Doc A/AC.105/C.1/2012/CRP.16 27 January 2101- Vienna 6,-17 February 2012 Item 14 of the draft provisional agenda, (2009); Asamblea General, Nota de la Secretaria- Documento AVAC.105/951, Investigaciones Nacionales sobre los desechos espaciales, la seguridad de los objetos espaciales con fuentes de energía nuclear a bordo y los problemas relativos a la colisión de esos objetos con desechos espaciales , 10 de Diciembre 2009

10. Neil McAleer (1992):"Arthur C. Clarke: The Authorized Biography", Contemporary Books, Chicago, 1992. ISBN 0-8092-37202

11. Piña, José $M^{a}$ (2008):“Basura Espacial y Satélites en Órbita, en "A día de hoy" 07/02/2008 en http://www.astropractica.org/ tact/basesp/basesp.htm 